University of Warwick institutional repository

This paper is made available online in accordance with

publisher policies. Please scroll down to view the document

itself. Please refer to the repository record for this item and our

policy information available from the repository home page for

further information.

To see the final version of this paper please visit the publisher's website. Access to the published version may require a subscription.

Author(s): Klaus Deckelnick, Charles M. Elliott, and Vanessa Styles

Article Title: Optimal Control of the Propagation of a Graph in Inhomogeneous Media Optimal Control of the Propagation of a Graph in Inhomogeneous Media

Year of publication: 2009

Link to published version : http://dx.doi.org/10.1137/080723648

Publisher statement: none 


\title{
OPTIMAL CONTROL OF THE PROPAGATION OF A GRAPH IN INHOMOGENEOUS MEDIA*
}

\author{
KLAUS DECKELNICK ${ }^{\dagger}$, CHARLES M. ELLIOTT ${ }^{\ddagger}$, AND VANESSA STYLES S $^{\S}$
}

\begin{abstract}
We study an optimal control problem for viscosity solutions of a Hamilton-Jacobi equation describing the propagation of a one-dimensional graph with the control being the speed function. The existence of an optimal control is proved together with an approximate controllability result in the $H^{-1}$-norm. We prove convergence of a discrete optimal control problem based on a monotone finite difference scheme and describe some numerical results.
\end{abstract}

Key words. optimal control, eikonal equation, Hamilton-Jacobi equation, approximate controllability

AMS subject classifications. 49J20, 49L25, 49M25

DOI. $10.1137 / 080723648$

1. Introduction. We are concerned with the formulation and numerical approximation of an optimal control problem for first order quasi-linear equations of Hamilton-Jacobi type describing the motion of a front in an inhomogeneous medium. Thus we are concerned with the initial value problem

$$
\begin{aligned}
V_{\Gamma} & =a, \\
\Gamma(0) & =\Gamma_{0},
\end{aligned}
$$

where $V_{\Gamma}$ denotes the normal velocity of an evolving surface $\Gamma(t) \in \mathbb{R}^{n+1}$ from an initial surface $\Gamma_{0}$. The strictly positive velocity function $a$ is taken to be spatially dependent. It is known for such an initial value problem that when it is formulated as finding $\Phi(x, t)$ satisfying

$$
\Phi_{t}=a|\nabla \Phi|, \quad \Phi(x, 0)=\Phi_{0}(x)
$$

and $\Gamma(t)$ is the zero level set of $\Phi(\cdot, t)$ there is a unique viscosity solution.

Such a problem arises in etching out a surface $\Gamma(t)$ with a prescribed etch rate which may depend on the medium $[12,3,2]$. It is natural to control the final surface at time $T$, say, using the etch rate. Suppose that the surface can be written as a graph in the plane, i.e.,

$$
\Gamma(t)=\{(x, y(x, t)) \mid x \in \mathbb{R}\} \subset \mathbb{R}^{2} .
$$

The issue is the control of the location of the graph at a given time evolving from a planar surface by choice of the prescribed velocity. We suppose that the speed function

\footnotetext{
* Received by the editors May 8, 2008; accepted for publication (in revised form) January 8, 2009; published electronically April 15, 2009.

http://www.siam.org/journals/sicon/48-3/72364.html

†Institut für Analysis und Numerik, Otto-von-Guericke-Universität Magdeburg, Universitätsplatz 2, 39106 Magdeburg, Germany (klaus.deckelnick@mathematik.uni-magdeburg.de).

${ }^{\ddagger}$ Mathematics Institute, University of Warwick, Coventry CV4 7AL, UK (c.m.elliott@warwick. ac.uk).

$\S$ Department of Mathematics, University of Sussex, Brighton BN1 9RF, UK (v.styles@sussex. ac.uk).
} 
is positive and depends only on the horizontal direction so that $a=a(x)>0$. Then (1.1) takes the form

$$
y_{t}=a(x) \sqrt{1+y_{x}^{2}}, \quad x \in \mathbb{R}, \quad t \in(0, T) .
$$

We have in mind the situation in which the etching process takes place in a finite time interval $(0, T)$ and in which the etching rate can be controlled. One situation would be where the medium is homogeneous but the etching rate can be controlled to be a space dependent function, whilst another situation would be where the medium is vertically striated so that the action of the etching is again space dependent. An example would be a vertically layered medium in which the etch speed is piecewise constant. We wish to fix the speed function in order that a particular etched shape is achieved at a fixed time $T$. Actually by scaling the time scale in the equation we can choose this time to be $T=1$.

The setting is that of a control problem for a first order quasi-linear partial differential equation of Hamilton-Jacobi type. In general it is known that optimal control for nonlinear hyperbolic equations is a difficult topic. Solutions of our state equation are considered in the context of viscosity solutions. In section 2 we formulate this as a control problem with a quadratic objective function and the graph eikonal equation as the state equation. The idea is that we seek the speed function $a$ to minimize the quadratic energy functional $\mathcal{J}(a)$, where

$$
\mathcal{J}(a)=\frac{1}{2} \int_{I}\left|y_{a}(x, T)-y_{T}(x)\right|^{2} d x+\delta \int_{I} a_{x}^{2} d x
$$

under certain constraints on $a$ where $y_{T}$ is the graph of the target etch shape, $y_{a}$ is the solution of the state equation, and $\delta \geq 0$. The first term of the energy is a fidelity term, whereas the second is a regularization for the speed in order to ensure that the state equation is well posed. If we allow the speed to depend only on a finite number of parameters, this term can be omitted. Let us note that a related problem involving a stationary eikonal equation for the first arrival time of a front was studied numerically in [11]. We also refer the reader to $[13,4]$ for results concerning the optimal control of first order conservation equations.

We begin by proving the existence of optimal controls for differing assumptions concerning the speed function. Since the solutions of the state equation are nonsmooth and nonunique, in general we need to consider viscosity solutions of the state equation. It is classical that there exists a unique viscosity solution when the speed function is continuous. When considering optimal control in this case one can apply $W^{1, \infty}$ constraints on the set of admissible functions which then would need to be applied when applying iterative descent methods for the optimization. This can be avoided by regularizing the functional (1.4) by adding a quadratic term in the gradient, that is, taking $\delta$ positive. On the other hand it is also interesting to consider discontinuous piecewise constant $a$, and this is the main focus of the paper. In this case we use the well-posedness theory of $[7,8]$ in order to prove the existence of an optimal control. An interesting question is whether the target is achievable. In general this will not be the case because the solution of the state equation for the speed function satisfying $\alpha \leq a(\cdot) \leq \beta$ has the global Lipschitz bound $\left\|\left(y_{a}\right)_{x}\right\|_{\infty} \leq \sqrt{\left(\frac{\beta}{\alpha}\right)^{2}-1}$. However, our next contribution is to show approximate controllability for $y_{T} \in C^{0}(I)$ by showing that there is a piecewise constant speed function $y_{a^{*}}$ such that the difference in $\left(H^{1}\right)^{\prime}$ between $y_{T}$ and $y_{a}^{*}$ is bounded in terms of the maximum width of the subintervals on which the speed can take differing constant values. 
In order to realize the optimal control we turn to numerical discretization. The idea is to discretize the state equation and the functional in such a way that the discrete optimization problem has a minimizer. This leaves the interesting and difficult question as to whether the discrete minimizers approximate a minimizer of the control problem. Our next result is to show convergence as the mesh size converges to zero. This relies on the use of monotone finite difference schemes for the Hamilton-Jacobi equation. We formulate this in the case of the discontinuous piecewise constant speed function. We choose to implement monotone finite difference schemes which are differentiable with respect to the solution so that the discrete minimization problem can be solved iteratively by a descent method based on a discrete adjoint equation. We found that this method worked very well in practice.

Our paper is organized as follows. Section 2 discusses existence and uniqueness of the state equation together with an existence result for the optimal control. An approximate controllability result in the $\left(H^{1}\right)^{\prime}$-norm is proved in section 2.2 . The numerical solution is considered in section 3 with a convergence result being proved for a monotone discretization of the state equation. We conclude with some numerical results.

\section{Formulation and existence.}

2.1. Mathematical setting. Let $I=[-1,1]$ and $0<\alpha<\beta<\infty$. We shall assume that the control function $a$ belongs to one of the following sets:

(K1)

$$
\begin{gathered}
K:=\left\{a: I \rightarrow \mathbb{R} \mid a(x)=a_{i}, x \in\left(\hat{x}_{i-1}, \hat{x}_{i}\right), a\left(\hat{x}_{i}\right)=\frac{a_{i}+a_{i+1}}{2}, i=1, \ldots, L-1,\right. \\
\left.a(-1)=a(1)=\frac{a_{1}+a_{L}}{2}, \alpha \leq a(x) \leq \beta, x \in I\right\},
\end{gathered}
$$

where $-1=\hat{x}_{0}<\hat{x}_{1}<\cdots<\hat{x}_{L-1}<\hat{x}_{L}=1$ is a partition of $I$.

$(\mathrm{K} 2) K:=\left\{a: I \rightarrow \mathbb{R} \mid a(x)=\sum_{i=1}^{L} a_{i} \phi_{i}(x), \alpha \leq a(x) \leq \beta, x \in I\right\}$, where $\left\{\phi_{i}\right\}_{i=1}^{L}$ satisfies $\phi_{i} \in W^{1, \infty}(I), \phi_{i}(-1)=\phi_{i}(1), \phi_{i}(x) \geq 0, i=1, \ldots, L$, and $\sum_{i=1}^{L} \phi_{i}(x)=1, x \in I$.

(K3) $K:=\left\{a \in W^{1,2}(I) \mid \alpha \leq a(x) \leq \beta, x \in I, a(-1)=a(1)\right\}$.

In what follows we think of the function $a$ as being extended to a 2-periodic function on $\mathbb{R}$. Given $a \in K$ we then consider the eikonal equation

$$
\begin{aligned}
y_{t} & =a(x) \sqrt{1+y_{x}^{2}} & & \text { in } \mathbb{R} \times(0, T], \\
y(\cdot, 0) & =0 & & \text { in } \mathbb{R}, \\
y(x, t) & =y(x+2, t), & & x \in \mathbb{R}, \quad 0 \leq t \leq T .
\end{aligned}
$$

According to Theorem 2.4 in section 2.2 the initial value problem (2.1)-(2.3) has a unique solution $y \in W^{1, \infty}(\mathbb{R} \times(0, T))$ which we shall denote by $y_{a}$ in order to indicate its dependence on $a \in K$. Let us now consider the following control problems:

- $(\mathrm{P}) \min \mathcal{J}(a)=\frac{1}{2} \int_{I}\left|y_{a}(x, T)-y_{T}(x)\right|^{2} d x$ subject to $a \in K$, where $K$ is given either by (K1) or (K2).

- $\left(\mathrm{P}^{\delta}\right) \min \mathcal{J}(a)=\frac{1}{2} \int_{I}\left|y_{a}(x, T)-y_{T}(x)\right|^{2} d x+\delta \int_{I} a_{x}^{2} d x$ subject to $a \in K$, where $K$ is the set given in (K3).

Here, $y_{T} \in L^{2}(I)$ is a given function and $\delta>0$.

Remark 2.1. In the definition of (K1) the choice of the values of $a$ at the end points of the intervals can be replaced by $a\left(\hat{x}_{i}\right)=a_{i}^{*}$, where $a_{i}^{*}$ is any value in the 
interval $\left[\min \left(a_{i}, a_{i+1}\right), \max \left(a_{i}, a_{i+1}\right)\right]$. This will not change the viscosity solution of the eikonal equation.

Remark 2.2. Although we have chosen to consider periodic boundary conditions we expect that our results can be extended to the situation of applying homogeneous Neumann boundary conditions for the eikonal equation.

2.2. Existence and uniqueness for the state equation. Let us begin by recalling the notion of viscosity solution originally introduced by Crandall and Lions [5] (see also [6]). Note that we pose the problem on all of $\mathbb{R}$, but since the initial data and the speed are periodic the resulting unique viscosity solution is also periodic. Since the function $a$ is possibly discontinuous we use a generalized definition due to Ishii [9]. Applied to our situation this results in the following.

DEFINITION 2.3. A function $y \in C^{0}(\mathbb{R} \times(0, T])$ is called a viscosity subsolution of (2.1) if for each $\zeta \in C^{\infty}(\mathbb{R} \times(0, \infty))$ the following holds: if $y-\zeta$ has a local maximum at a point $\left(x_{0}, t_{0}\right) \in \mathbb{R} \times(0, T]$, then

$$
\zeta_{t}\left(x_{0}, t_{0}\right) \leq a^{*}\left(x_{0}\right) \sqrt{1+\zeta_{x}\left(x_{0}, t_{0}\right)^{2}} .
$$

A function $y \in C^{0}(\mathbb{R} \times(0, T])$ is called a viscosity supersolution of (2.1) if for each $\zeta \in C^{\infty}(\mathbb{R} \times(0, \infty))$ the following holds: if $y-\zeta$ has a local minimum at a point $\left(x_{0}, t_{0}\right) \in \mathbb{R} \times(0, T]$, then

$$
\zeta_{t}\left(x_{0}, t_{0}\right) \geq a_{*}\left(x_{0}\right) \sqrt{1+\zeta_{x}\left(x_{0}, t_{0}\right)^{2}} .
$$

A viscosity solution of $(2.1),(2.2)$ is then a function $y \in C^{0}(\mathbb{R} \times[0, T])$ which is both $a$ viscosity sub-and supersolution and which satisfies $y(x, 0)=0$ for all $x \in \mathbb{R}$.

In the above,

$$
\begin{aligned}
& a^{*}(x):=\lim _{r \rightarrow 0} \sup \{a(y)|| x-y \mid<r\}, \\
& a_{*}(x):=\lim _{r \rightarrow 0} \inf \{a(y)|| x-y \mid<r\}
\end{aligned}
$$

denote the upper and lower semicontinuous envelopes of $a$, respectively.

TheOrem 2.4. Let $a \in K$, where $K$ is one of the sets given in (K1)-(K3). Then there exists a unique viscosity solution $y \in W^{1, \infty}(\mathbb{R} \times[0, T])$ of $(2.1)-(2.3)$ and $y$ satisfies

$$
\|y\|_{W^{1, \infty}(\mathbb{R} \times[0, T])} \leq C,
$$

where $C$ depends on $T, \alpha$, and $\beta$.

Proof. We will give the main ideas of a proof which covers the cases (K1)-(K3).

Existence. Consider the following regularized problem:

$$
\begin{aligned}
y_{t}^{\epsilon}-\epsilon \frac{y_{x x}^{\epsilon}}{1+\left(y_{x}^{\epsilon}\right)^{2}} & =a^{\epsilon} \sqrt{1+\left(y_{x}^{\epsilon}\right)^{2}} \quad \text { in } \mathbb{R} \times(0, T], \\
y^{\epsilon}(\cdot, 0) & =0 \quad \text { in } \mathbb{R}, \\
y^{\epsilon}(x+2, t) & =y^{\epsilon}(x, t), \quad x \in \mathbb{R}, \quad 0<t \leq T .
\end{aligned}
$$

Here, $\epsilon>0$ and $a^{\epsilon}$ is a suitable mollification of $a$ with

$$
\alpha \leq a^{\epsilon}(x) \leq \beta, \quad a^{\epsilon}(x+2)=a^{\epsilon}(x) \quad \text { for all } x \in \mathbb{R} .
$$


Using the Leray-Schauder principle it is possible to prove that (2.5)-(2.7) has a unique smooth solution [10]. The corresponding argument relies on the derivation of a priori estimates on $y_{t}^{\epsilon}$ and $y_{x}^{\epsilon}$ for a solution of (2.5)-(2.7). As these bounds also motivate why (2.4) holds we shall briefly sketch their proof for the convenience of the reader. Differentiating (2.5) with respect to time gives

$$
y_{t t}^{\epsilon}-\epsilon \frac{y_{t, x x}^{\epsilon}}{1+\left(y_{x}^{\epsilon}\right)^{2}}+2 \epsilon \frac{y_{x x}^{\epsilon} y_{x}^{\epsilon}}{\left(1+\left(y_{x}^{\epsilon}\right)^{2}\right)^{2}} y_{t, x}^{\epsilon}-a^{\epsilon} \frac{y_{x}^{\epsilon}}{\sqrt{1+\left(y_{x}^{\epsilon}\right)^{2}}} y_{t, x}^{\epsilon}=0 \quad \text { in } \mathbb{R} \times(0, T) .
$$

Since $y_{t}^{\epsilon}$ is a bounded solution of a parabolic equation of the form $\phi_{t}-a_{\epsilon} \phi_{x x}+b_{\epsilon} \phi_{x}=0$ on $\mathbb{R} \times(0, T]$, the maximum principle together with (2.6) and (2.8) implies

$$
\max _{\mathbb{R} \times[0, T]}\left|y_{t}^{\epsilon}\right|=\max _{\mathbb{R}}\left|y_{t}^{\epsilon}(\cdot, 0)\right|=\max _{\mathbb{R}} a^{\epsilon} \leq \beta .
$$

In order to derive an estimate on $y_{x}^{\epsilon}$ we introduce $z(x, t):=\left(y_{x}^{\epsilon}(x, t)\right)^{2}$. We can assume that $\mu:=\max _{I \times[0, T]} z>0$. There exists a point $\left(x_{0}, t_{0}\right) \in I \times[0, T]$ such that $z\left(x_{0}, t_{0}\right)=\mu$. Then $z_{x}\left(x_{0}, t_{0}\right)=0$, which is also true in the case $x_{0} \in \partial I$ by the periodicity of $z$. As a result, $y_{x}^{\epsilon}\left(x_{0}, t_{0}\right) y_{x x}^{\epsilon}\left(x_{0}, t_{0}\right)=0$, from which we infer that $y_{x x}^{\epsilon}\left(x_{0}, t_{0}\right)=0$ since $y_{x}^{\epsilon}\left(x_{0}, t_{0}\right) \neq 0$ in view of $\mu>0$. Substituting this information into (2.5) gives

$$
y_{t}^{\epsilon}\left(x_{0}, t_{0}\right)=a^{\epsilon}\left(x_{0}\right) \sqrt{1+z\left(x_{0}, t_{0}\right)},
$$

and therefore by (2.9) and (2.8)

$$
\max _{I \times[0, T]}\left|y_{x}^{\epsilon}\right|=z\left(x_{0}, t_{0}\right)^{\frac{1}{2}} \leq \sqrt{\frac{y_{t}^{\epsilon}\left(x_{0}, t_{0}\right)^{2}}{a^{\epsilon}\left(x_{0}\right)^{2}}-1} \leq \sqrt{\left(\frac{\beta}{\alpha}\right)^{2}-1} .
$$

Since the estimates (2.9) and (2.10) are uniform in $\epsilon$ there exist a subsequence $\epsilon_{j} \searrow 0$ and a function $y \in W^{1, \infty}(\mathbb{R} \times[0, T])$, which is 2-periodic in space such that

$$
y^{\epsilon_{j}} \rightarrow y \quad \text { uniformly in } \mathbb{R} \times[0, T] .
$$

It can be shown that $y$ is a viscosity solution of (2.1)-(2.3) (see [6] if $a$ is continuous and, e.g., $[7,8]$ for the case of discontinuous $a)$.

Uniqueness. In the case of a continuous speed function a uniqueness result for (2.1) can be found in [6]. A comparison and uniqueness result for a discontinuous speed function is obtained in section 3 of [8]. The theory developed in [8] requires three conditions (A1)-(A3) on the function $a$ which appear on page 1164. An inspection of the calculations in section 3 shows that only (A2) and (A3) are actually needed to obtain comparison and uniqueness. It is easily seen that these two conditions are satisfied in our case; cf. also Remark 2 (1) on page 1165.

Corollary 2.5. Suppose that $a, \tilde{a} \in K$ with $a \leq \tilde{a}$ a.e. in I. Then

$$
y_{a} \leq y_{\tilde{a}} \quad \text { in } \mathbb{R} \times[0, T] .
$$

Proof. Let us denote by $y^{\epsilon}$ and $\tilde{y}^{\epsilon}$ the solutions of (2.5)-(2.7) corresponding to $a^{\epsilon}, \tilde{a}^{\epsilon}$, respectively. Here, $a^{\epsilon}, \tilde{a}^{\epsilon}$ are mollifications of $a, \tilde{a}$ so that $a^{\epsilon} \leq \tilde{a}^{\epsilon}$ in $\mathbb{R}$. Recalling the proof of Theorem 2.4 we may assume that for some sequence $\epsilon_{j} \searrow 0, j \rightarrow \infty$

$$
y^{\epsilon_{j}} \rightarrow y_{a}, \quad \tilde{y}^{\epsilon_{j}} \rightarrow y_{\tilde{a}} \quad \text { uniformly in } \mathbb{R} \times[0, T] .
$$

Copyright (c) by SIAM. Unauthorized reproduction of this article is prohibited. 
The functions $v^{j}:=y^{\epsilon_{j}}-\tilde{y}^{\epsilon_{j}}$ are 2-periodic in space and satisfy a differential inequality of the form

$$
v_{t}^{j}-b^{j} v_{x x}^{j}+c^{j} v_{x}^{j} \leq 0 \quad \text { in } \mathbb{R} \times[0, T]
$$

for suitable functions $c^{j}$ and $b^{j}>0$. Since $v^{j}(\cdot, 0)=0$ the maximum principle implies that $v^{j} \leq 0$ in $\mathbb{R} \times[0, T]$, and the result follows after sending $j \rightarrow \infty$.

Corollary 2.6. Let $K$ be defined by (K1) and suppose that $a \in K$ satisfies $a_{k}=\beta$ for some $k \in\{1, \ldots, L\}$. Then

$$
y_{a}(x, t)=\beta t, \quad x \in\left[\hat{x}_{k-1}, \hat{x}_{k}\right], \quad t>0 .
$$

Proof. Define $\tilde{a} \in K$ by $\tilde{a}_{i}=\beta, i=1, \ldots, L$. Clearly, $y_{\tilde{a}} \equiv \beta t$ so that Corollary 2.5 implies that $y_{a}(x, t) \leq \beta t, x \in \mathbb{R}, t>0$. Let $a^{\epsilon}$ and $y^{\epsilon}$ be as in the proof of Theorem 2.4, so that

$$
a^{\epsilon_{n}} \rightarrow a \text { a.e. in } I, \quad y^{\epsilon_{n}} \rightarrow y_{a} \text { uniformly in } \mathbb{R} \times[0, T]
$$

for some suitable sequence $\left(\epsilon_{n}\right)_{n \in \mathbb{N}}$ with $\epsilon_{n} \searrow 0, n \rightarrow \infty$. Recalling (2.6) and (2.5) we obtain for $t \in(0, T]$

$$
\begin{aligned}
& \int_{\hat{x}_{k-1}}^{\hat{x}_{k}}\left|\beta t-y_{a}(\cdot, t)\right|=\int_{\hat{x}_{k-1}}^{\hat{x}_{k}}\left(\beta t-y_{a}(\cdot, t)\right)=\lim _{n \rightarrow \infty} \int_{\hat{x}_{k-1}}^{\hat{x}_{k}}\left(a^{\epsilon_{n}} t-y^{\epsilon_{n}}(\cdot, t)\right) \\
& =\lim _{n \rightarrow \infty} \int_{0}^{t} \int_{\hat{x}_{k-1}}^{\hat{x}_{k}}\left(a^{\epsilon_{n}}-y_{t}^{\epsilon_{n}}\right) \leq \varlimsup_{n \rightarrow \infty}^{t} \int_{0}^{t} \int_{\hat{x}_{k-1}}^{\hat{x}_{k}}\left(a^{\epsilon_{n}} \sqrt{1+\left(y_{x}^{\epsilon_{n}}\right)^{2}}-y_{t}^{\epsilon_{n}}\right) \\
& =\varlimsup_{n \rightarrow \infty}\left(\left(-\epsilon_{n}\right) \int_{0}^{t} \int_{\hat{x}_{k-1}}^{\hat{x}_{k}} \frac{y_{x x}^{\epsilon_{n}}}{1+\left(y_{x}^{\epsilon_{n}}\right)^{2}}\right) \\
& =\varlimsup_{n \rightarrow \infty}\left(\left.\left(-\epsilon_{n}\right) \int_{0}^{t} \arctan \left(y_{x}^{\epsilon_{n}}\right)\right|_{\hat{x}_{k-1}} ^{\hat{x}_{k}}\right) \rightarrow 0, n \rightarrow \infty,
\end{aligned}
$$

which implies the desired result.

\subsection{Existence for the optimal control problem.}

TheOREM 2.7. For $y_{T} \in L^{2}(I)$ the control problems $(\mathrm{P})$ and $\left(\mathrm{P}^{\delta}\right)$ have at least one solution $a \in K$.

Proof. Let us first consider (P) and suppose that $\left(a^{m}\right)_{m \in \mathbb{N}} \subset K$ is a minimizing sequence so that

$$
\mathcal{J}\left(a^{m}\right) \searrow \inf _{a \in K} \mathcal{J}(a) \quad \text { as } m \rightarrow \infty .
$$

The corresponding solutions $y^{m}=y_{a^{m}}$ of the state equation satisfy in view of (2.4)

$$
\left\|y^{m}\right\|_{W^{1, \infty}(\mathbb{R} \times[0, T])} \leq C=C(T, \alpha, \beta) .
$$

Combining this estimate with the observation that for (K1), (K2) the set $K$ is contained in a finite-dimensional space we obtain a subsequence $\left(m_{k}\right)_{k \in \mathbb{N}}$ and functions $a \in K, y \in W^{1, \infty}(\mathbb{R} \times[0, T])$ such that

$$
a^{m_{k}} \rightarrow a \quad \text { uniformly in } I, \quad y^{m_{k}} \rightarrow y \quad \text { uniformly in } \mathbb{R} \times[0, T] .
$$

We claim that $y=y_{a}$. We show first that $y$ is a viscosity subsolution in the sense of Definition 2.3. Let $\zeta \in C^{\infty}(\mathbb{R} \times(0, \infty))$ and suppose that $y-\zeta$ has a local maximum 
at some point $\left(x_{0}, t_{0}\right) \in \mathbb{R} \times(0, T]$. Using standard arguments we can assume that $\left(x_{0}, t_{0}\right)$ is a strict maximum from which we deduce the existence of a sequence $\left(x_{k}, t_{k}\right)$ with the properties that

$$
\lim _{k \rightarrow \infty}\left(x_{k}, t_{k}\right)=\left(x_{0}, t_{0}\right) \quad \text { and } \quad y^{m_{k}}-\zeta \text { has a local maximum at }\left(x_{k}, t_{k}\right) .
$$

Since $y^{m_{k}}$ is a viscosity solution of (2.1) we infer that

$$
\zeta_{t}\left(x_{k}, t_{k}\right) \leq\left(a^{m_{k}}\right)^{*}\left(x_{k}\right) \sqrt{1+\left(\zeta_{x}\left(x_{k}, t_{k}\right)\right)^{2}} .
$$

Observing that

$$
\lim \sup _{k \rightarrow \infty}\left(a^{m_{k}}\right)^{*}\left(x_{k}\right) \leq a^{*}\left(x_{0}\right)
$$

we obtain after passing to the limit $k \rightarrow \infty$

$$
\zeta_{t}\left(x_{0}, t_{0}\right) \leq a^{*}\left(x_{0}\right) \sqrt{1+\left(\zeta_{x}\left(x_{0}, t_{0}\right)\right)^{2}} .
$$

In a similar way one proves that $y$ is a viscosity supersolution, so that $y=y_{a}$. Finally we infer from the uniform convergence of $y^{m_{k}}$ to $y$ that $\mathcal{J}(a)=\inf _{\tilde{a} \in K} \mathcal{J}(\tilde{a})$.

In the case of $\left(\mathrm{P}^{\delta}\right)$ where $K$ is given as in (K3) we observe that a minimizing sequence $a^{m}$ is bounded uniformly in $H^{1}(I)$ so that there is a subsequence $\left(m_{k}\right)_{k \in \mathbb{N}}$ and $a \in K$ such that

$$
a^{m_{k}} \rightarrow a \quad \text { uniformly in } I, \quad a_{x}^{m_{k}} \rightarrow a_{x} \quad \text { in } L^{2}(I) .
$$

The remainder of the proof follows in a fashion similar to the previous case.

3. Approximate controllability in the $\left(H^{1}\right)^{\prime}$-norm. Let us consider problem (P) for a given target $y_{T} \in C^{0}(I)$. We assume that the set $K$ is given by (K1), so that the admissible control functions are constant on $I_{i}=\left(\hat{x}_{i-1}, \hat{x}_{i}\right)$, where $-1=\hat{x}_{0}<\hat{x}_{1}<\cdots<\hat{x}_{L-1}<\hat{x}_{L}=1$ is a partition of $I$. It is natural to expect that $y_{T}$ can be reached in a better way as we decrease the fineness of the partition. It turns out that this can be made mathematically precise if one works in a weak norm and allows the speeds to become small but still positive. In what follows we fix the upper bound $\beta$ on the speeds in such a way that

$$
\beta>\frac{1}{T} \max _{x \in I} y_{T}(x) \text {. }
$$

Let us introduce the set

$$
\begin{gathered}
K_{\beta}:=\left\{a: I \rightarrow \mathbb{R} \mid a(x)=a_{i}, x \in\left(\hat{x}_{i-1}, \hat{x}_{i}\right), a\left(\hat{x}_{i}\right)=\frac{a_{i}+a_{i+1}}{2}, i=1, \ldots, L-1,\right. \\
\left.a(-1)=a(1)=\frac{a_{1}+a_{L}}{2}, 0<a_{i} \leq \beta, i=1, \ldots, L\right\} .
\end{gathered}
$$

The following lemma says that if the speed is sufficiently small on some subinterval $I_{i}$, then the mean value of the corresponding solution over $I_{i}$ will lie below $\min _{x \in I} y_{T}(x)$.

Lemma 3.1. Let $y_{T} \in C^{0}(I)$ be positive. Then there exists $0<\alpha<\frac{1}{T} \min _{x \in I} y_{T}(x)$ such that for every $a \in K_{\beta}$ the following holds: if $a_{i}=\tilde{\alpha}$ for some $i \in\{1, \ldots, L\}$ and $\tilde{\alpha} \leq \alpha$, then $\frac{1}{\left|I_{i}\right|} \int_{I_{i}} y_{a}(\cdot, T)<\min _{x \in I} y_{T}(x)$.

Proof. Let $a \in K_{\beta}$ and suppose that $a_{i}=\tilde{\alpha} \leq \alpha$ for some $i \in\{1, \ldots, L\}$, where $\alpha$ will be determined later. Corollary 2.5 implies that

$$
y_{a}(x, t) \leq y_{\hat{a}}(x, t), \quad(x, t) \in \mathbb{R} \times[0, \infty),
$$


where $\hat{a} \in K$ is given by $\hat{a}_{i}=\alpha$ and $\hat{a}_{k}=\beta$ for $k \neq i$. It is therefore sufficient to prove the claim for $\hat{a}$. One checks that $y_{\hat{a}}$ is given on $I \times[0, T]$ by the formula

$$
y_{\hat{a}}(x, t)= \begin{cases}\beta t, & \hat{x}_{k-1} \leq x \leq \hat{x}_{k}, \quad k \neq i, \\ \beta t-\gamma\left(x-\hat{x}_{i-1}\right), & \hat{x}_{i-1} \leq x \leq \hat{x}_{i-1}+c t \\ \alpha t, & \hat{x}_{i-1}+c t \leq x \leq \hat{x}_{i}-c t, \\ \beta t+\gamma\left(x-\hat{x}_{i}\right), & \hat{x}_{i}-c t \leq x \leq \hat{x}_{i},\end{cases}
$$

where

$$
\gamma=\sqrt{\frac{\beta^{2}}{\alpha^{2}}-1} \quad \text { and } \quad c=\frac{\beta-\alpha}{\gamma} .
$$

Here, $\alpha$ is chosen a priori so small that $2 c T \leq \inf _{i}\left(\hat{x}_{i}-\hat{x}_{i-1}\right)$. A straightforward calculation shows that

$$
\begin{aligned}
\int_{I_{i}} y_{\hat{a}}(\cdot, T) & =2(\beta-\alpha) c T^{2}-\gamma c^{2} T^{2}+\alpha\left(\hat{x}_{i}-\hat{x}_{i-1}\right) T \\
& =\gamma c^{2} T^{2}+\alpha\left|I_{i}\right| T=\frac{\alpha(\beta-\alpha)^{\frac{3}{2}} T^{2}}{(\beta+\alpha)^{\frac{1}{2}}}+\alpha\left|I_{i}\right| T \leq \alpha \beta T^{2}+\alpha\left|I_{i}\right| T .
\end{aligned}
$$

Hence we have that $\frac{1}{\left|I_{i}\right|} \int_{I_{i}} y_{a}(\cdot, T)<\min _{x \in I} y_{T}(x)$, provided that

$$
\frac{\alpha \beta T}{\left|I_{i}\right|}+\alpha<\frac{1}{T} \min _{x \in I} y_{T}(x),
$$

which can be achieved for $\alpha>0$ sufficiently small.

We can now formulate the main result of this section.

Theorem 3.2. Let $y_{T} \in C^{0}(I)$ be positive and $-1=\hat{x}_{0}<\hat{x}_{1}<\cdots<\hat{x}_{L-1}<$ $\hat{x}_{L}=1$. Then

$$
\inf _{a \in K_{\beta}}\left\|y_{a}(\cdot, T)-y_{T}\right\|_{\left(H^{1}\right)^{\prime}} \leq\left(\sqrt{2} \beta T+\left\|y_{T}\right\|_{L^{2}(I)}\right) \max _{i=1, \ldots, L}\left(\hat{x}_{i}-\hat{x}_{i-1}\right) .
$$

Proof. Choose $0<\alpha<\frac{1}{T} \min _{x \in I} y_{T}(x)$ according to Lemma 3.1 and set $\Omega:=(\alpha, \beta)^{L} \subset \mathbb{R}^{L}$. We define $F: \bar{\Omega} \rightarrow \mathbb{R}^{L}$ by

$$
F(\underline{a})_{i}:=\frac{1}{T\left|I_{i}\right|} \int_{I_{i}} y_{a}(\cdot, T), \quad i=1, \ldots, L
$$

where $\underline{a}=\left(a_{1}, \ldots, a_{L}\right)^{t}$ and $a: I \rightarrow \mathbb{R}$ is the corresponding piecewise constant function. Using arguments similar to those in section 2 one can show that $F$ is continuous. Let $\underline{b} \in \mathbb{R}^{L}$ be given by

$$
b_{i}=\frac{1}{T\left|I_{i}\right|} \int_{I_{i}} y_{T}, \quad i=1, \ldots, L
$$

Recalling (3.1) we have

$$
\alpha<\frac{1}{T} \min _{x \in I} y_{T}(x) \leq b_{i} \leq \frac{1}{T} \max _{x \in I} y_{T}(x)<\beta, \quad i=1, \ldots, L,
$$

so that $\underline{b} \in \Omega$. We now consider the following homotopy $H: \bar{\Omega} \times[0,1] \rightarrow \mathbb{R}^{L}$ :

$$
H(\underline{a}, \sigma):=\sigma F(\underline{a})+(1-\sigma) \underline{a}-\underline{b} .
$$

Copyright (c) by SIAM. Unauthorized reproduction of this article is prohibited. 
Denoting by deg the Brouwer degree we have

$$
\operatorname{deg}(H(\cdot, 0), \Omega, 0)=\operatorname{deg}(\operatorname{id}-\underline{b}, \Omega, 0)=\operatorname{deg}(\operatorname{id}, \Omega, \underline{b})=1,
$$

since $\underline{b} \in \Omega$. In order to verify that $H$ is an admissible homotopy we have to show that $0 \notin H(\partial \Omega, \sigma)$ for all $\sigma \in(0,1]$. Assume that there exist $\underline{a} \in \partial \Omega$ and $\sigma \in(0,1]$ such that $H(\underline{a}, \sigma)=0$, i.e.,

$$
b_{i}=\sigma \frac{1}{T\left|I_{i}\right|} \int_{I_{i}} y_{\underline{a}}(\cdot, T)+(1-\sigma) a_{i}, \quad i=1, \ldots, L .
$$

We distinguish two cases.

Case 1. $a_{i}=\beta$ for some $i \in\{1, \ldots, L\}$. Corollary 2.6 implies that $y_{a}(x, T)=$ $\beta T, x \in I_{i}$ so that (3.3) yields $b_{i}=\beta$, a contradiction to (3.2).

Case 2. $a_{i}=\alpha$ for some $i \in\{1, \ldots, L\}$. In this case we conclude with the help of (3.3) and Lemma 3.1 that

$$
b_{i}=\sigma \frac{1}{T\left|I_{i}\right|} \int_{I_{i}} y_{\underline{a}}(\cdot, T)+(1-\sigma) \alpha<\sigma \frac{1}{T} \min _{x \in I} y_{T}(x)+(1-\sigma) \frac{1}{T} \min _{x \in I} y_{T}(x) \leq b_{i},
$$

which is again a contradiction. As a result, $H$ is an admissible homotopy so that $\operatorname{deg}(H(\cdot, 1), \Omega, 0)=1$ and by the properties of the Brouwer degree there exists $\underline{a} \in \Omega$ such that $H(\underline{a}, 1)=0$, or equivalently

$$
\int_{I_{i}} y_{\underline{a}}(\cdot, T)=\int_{I_{i}} y_{T}, \quad i=1, \ldots, L
$$

Let $\varphi \in H^{1}(I)$. For the state corresponding to $\underline{a}$ we deduce that

$$
\int_{I}\left(y_{a}(\cdot, T)-y_{T}\right) \varphi=\sum_{i=1}^{L} \int_{I_{i}}\left(y_{a}(\cdot, T)-y_{T}\right) \varphi=\sum_{i=1}^{L} \int_{I_{i}}\left(y_{a}(\cdot, T)-y_{T}\right)\left(\varphi-\frac{1}{\left|I_{i}\right|} \int_{I_{i}} \varphi\right)
$$

by (3.4). Using Poincaré's inequality we deduce that

$$
\begin{aligned}
\left|\int_{I}\left(y_{a}(\cdot, T)-y_{T}\right) \varphi\right| & \leq \sum_{i=1}^{L}\left(\left\|y_{a}(\cdot, T)\right\|_{L^{2}\left(I_{i}\right)}+\left\|y_{T}\right\|_{L^{2}\left(I_{i}\right)}\right)\left(\hat{x}_{i}-\hat{x}_{i-1}\right)\left\|\varphi^{\prime}\right\|_{L^{2}\left(I_{i}\right)} \\
\leq & \max _{i=1, \ldots, L}\left(\hat{x}_{i}-\hat{x}_{i-1}\right)\left(\left\|y_{a}(\cdot, T)\right\|_{L^{2}(I)}+\left\|y_{T}\right\|_{L^{2}(I)}\right)\left\|\varphi^{\prime}\right\|_{L^{2}(I)} \\
\leq & \max _{i=1, \ldots, L}\left(\hat{x}_{i}-\hat{x}_{i-1}\right)\left(\sqrt{2}\left\|y_{a}(\cdot, T)\right\|_{L^{\infty}(I)}+\left\|y_{T}\right\|_{L^{2}(I)}\right)\left\|\varphi^{\prime}\right\|_{L^{2}(I)} .
\end{aligned}
$$

Since $0 \leq y_{a}(x, T) \leq \beta T, x \in I$, we obtain

$$
\left\|y_{a}(\cdot, T)-y_{T}\right\|_{\left(H^{1}\right)^{\prime}} \leq\left(\sqrt{2} \beta T+\left\|y_{T}\right\|_{L^{2}(I)}\right) \max _{i=1, \ldots, L}\left(\hat{x}_{i}-\hat{x}_{i-1}\right)
$$

and the theorem is proved.

4. Numerical approximation. Let us choose space and time steps $h$ and $\tau$, respectively, and let

$$
\begin{aligned}
& x_{j}=j h-1, \quad j \in \mathbb{Z}, \\
& t_{n}=n \tau, \quad 0 \leq n \leq N,
\end{aligned}
$$

Copyright (c) by SIAM. Unauthorized reproduction of this article is prohibited. 
where $h=2 / J$ and $\tau=T / N$. Furthermore, we set $G_{h, \tau}:=\left\{\left(x_{j}, t_{n}\right) \in \mathbb{R} \times[0, T] \mid j \in\right.$ $\mathbb{Z}, 0 \leq n \leq N\}$. For a grid function $Y: G_{h, \tau} \rightarrow \mathbb{R}$ we denote by $Y_{j}^{n}$ its value at the point $\left(x_{j}, t_{n}\right)$. It is convenient to define the difference operators

$$
\delta_{h}^{+} Y_{j}^{n}:=\frac{Y_{j+1}^{n}-Y_{j}^{n}}{h}, \quad \delta_{h}^{-} Y_{j}^{n}:=\frac{Y_{j}^{n}-Y_{j-1}^{n}}{h}, \quad \delta_{t} Y_{j}^{n}:=\frac{Y_{j}^{n+1}-Y_{j}^{n}}{\tau} .
$$

In what follows we aim to find approximate solutions of (P) where $K$ is given by (K1). We begin by discussing the discretization of the state equation.

4.1. Discretization of the state equation. The solution of the state equation (2.1)-(2.3) is approximated by the grid function $Y: G_{h, \tau} \rightarrow \mathbb{R}$ which for a given $a \in K$ satisfies

$$
\begin{aligned}
\delta_{t} Y_{j}^{n} & =a\left(x_{j}\right) \mathcal{F}\left(\delta_{h}^{+} Y_{j}^{n}, \delta_{h}^{-} Y_{j}^{n}\right), \quad j=1, \ldots, J, \quad n=0, \ldots, N-1, \\
Y_{j}^{0} & =0, \quad j \in \mathbb{Z}, \\
Y_{j+J}^{n} & =Y_{j}^{n}, \quad j \in \mathbb{Z}, \quad n=1, \ldots, N,
\end{aligned}
$$

where we have chosen the discrete Hamiltonian to be

$$
\mathcal{F}(p, q)=\sqrt{1+\left(p^{+}\right)^{2}}+\sqrt{1+\left(q^{-}\right)^{2}}-1 .
$$

Here, $p^{+}=\max (p, 0), q^{-}=\min (q, 0)$. The relation (4.2) can be written in the form

$$
Y^{n+1}=G\left(Y^{n}\right), \quad 0 \leq n \leq N-1,
$$

where

$G(V)_{j}:=V_{j}+\tau a\left(x_{j}\right) \mathcal{F}\left(\delta_{h}^{+} V_{j}, \delta_{h}^{-} V_{j}\right), \quad j=1, \ldots, J ; \quad G(V)_{j+J}=G(V)_{j}, \quad j \in \mathbb{Z}$,

for a grid function $V: \mathbb{R} / h \mathbb{Z} \rightarrow \mathbb{R}$.

Following [1] we need to show that (4.2)-(4.4) gives rise to a consistent, monotone and stable approximation of the state equation. The first point follows from the relation

$$
\mathcal{F}(p, p)=\sqrt{1+p^{2}}, \quad p \in \mathbb{R} .
$$

Let us next address the monotonicity of the scheme.

Lemma 4.1. Suppose that $\tau \leq \frac{h}{2 \beta}$. Then $G$ is monotone; i.e., $V \leq W$ implies that $G(V) \leq G(W)$.

Proof. Let $V \leq W$. Clearly,

$$
0 \leq \frac{\partial \mathcal{F}}{\partial p}(p, q) \leq 1, \quad-1 \leq \frac{\partial \mathcal{F}}{\partial q}(p, q) \leq 0, \quad p, q \in \mathbb{R} .
$$

As a consequence we have for $j=1, \ldots, J$

$$
\begin{aligned}
G(W)_{j}- & G(V)_{j} \\
= & W_{j}-V_{j}+\tau a\left(x_{j}\right)\left(\mathcal{F}\left(\delta_{h}^{+} W_{j}, \delta_{h}^{-} W_{j}\right)-\mathcal{F}\left(\delta_{h}^{+} V_{j}, \delta_{h}^{-} V_{j}\right)\right) \\
= & \left(W_{j}-V_{j}\right)\left(1-\frac{\tau}{h} \xi a\left(x_{j}\right)+\frac{\tau}{h} \eta a\left(x_{j}\right)\right) \\
& +\frac{\tau}{h} a\left(x_{j}\right)\left(\xi\left(W_{j+1}-V_{j+1}\right)-\eta\left(W_{j-1}-V_{j-1}\right)\right)
\end{aligned}
$$

Copyright (c) by SIAM. Unauthorized reproduction of this article is prohibited. 
for some $\xi \in[0,1], \eta \in[-1,0]$. Recalling that $a\left(x_{j}\right) \leq \beta, \tau \leq \frac{h}{2 \beta}$, and $W \geq V$ we deduce that $G(W)_{j} \geq G(V)_{j}$.

Finally we have the following stability bounds which reflect corresponding estimates for the continuous problem.

Corollary 4.2. Let $a \in K$ and suppose that $\tau \leq \frac{h}{2 \beta}$. Then the solution $Y$ of (4.2)-(4.4) satisfies

(a) $\quad 0 \leq \frac{Y_{j}^{n+1}-Y_{j}^{n}}{\tau} \leq \beta, \quad j \in \mathbb{Z}, \quad n=0, \ldots, N-1 ;$

(b) $\quad\left|\frac{Y_{j+1}^{n}-Y_{j}^{n}}{h}\right| \leq \frac{\beta}{\alpha}, \quad j \in \mathbb{Z}, \quad n=0, \ldots, N$.

Proof. The lower bound in (a) follows immediately from the definition of the scheme and the fact that $a$ and $\mathcal{F}$ are nonnegative. We prove the upper bound by induction on $n$. The assertion is clear for $n=0$. Let us suppose that it holds for some $n \in\{0, \ldots, N-2\}$. Then we have $Y_{j}^{n+1} \leq Y_{j}^{n}+\beta \tau, j \in \mathbb{Z}$, so that Lemma 4.1 together with the relation $G(V+c)_{j}=G(V)_{j}+c(c \in \mathbb{R})$ yields

$$
Y_{j}^{n+2}=G\left(Y^{n+1}\right)_{j} \leq G\left(Y^{n}\right)_{j}+\beta \tau=Y_{j}^{n+1}+\beta \tau .
$$

Since $\mathcal{F}(p, q) \geq \max \left(p^{+},\left|q^{-}\right|\right)$we deduce from (a) for $j \in \mathbb{Z}$ that

$$
\beta \geq \frac{Y_{j}^{n+1}-Y_{j}^{n}}{\tau}=a\left(x_{j}\right) \mathcal{F}\left(\delta_{h}^{+} Y_{j}^{n}, \delta_{h}^{-} Y_{j}^{n}\right) \geq \alpha \max \left(\left|\left(\delta_{h}^{+} Y_{j}^{n}\right)^{+}\right|,\left|\left(\delta_{h}^{-} Y_{j}^{n}\right)^{-}\right|\right) .
$$

Observing that $\delta_{h}^{+} Y_{j}^{n}=\delta_{h}^{-} Y_{j+1}^{n}$, (b) follows. via

We extend the solution $Y$ of (4.2)-(4.4) to a function $Y_{h, \tau} \in W^{1, \infty}(\mathbb{R} \times(0, T))$

$$
\begin{aligned}
Y_{h, \tau}(x, t):= & \frac{t_{n+1}-t}{\tau}\left(\frac{x_{j+1}-x}{h} Y_{j}^{n}+\frac{x-x_{j}}{h} Y_{j+1}^{n}\right) \\
& +\frac{t-t_{n}}{\tau}\left(\frac{x_{j+1}-x}{h} Y_{j}^{n+1}+\frac{x-x_{j}}{h} Y_{j+1}^{n+1}\right)
\end{aligned}
$$

if $(x, t) \in\left[x_{j}, x_{j+1}\right] \times\left[t_{n}, t_{n+1}\right]$. Note that $Y_{h, \tau}(x+2, t)=Y_{h, \tau}(x, t)$. Furthermore, Corollary 4.2 implies that

$$
\left\|Y_{h, \tau}\right\|_{W^{1, \infty}} \leq C(T, \alpha, \beta) \quad \text { for } \tau \leq \frac{h}{2 \beta} .
$$

We are now in position to prove a convergence result for the approximation of the state equation.

TheOrEm 4.3. Let $(h, \tau)$ denote a sequence such that $\tau \leq \frac{h}{2 \beta}, h \rightarrow 0$, and let $Y_{h, \tau}$ be the corresponding solutions of (4.2)-(4.4). Then $Y_{h, \tau} \rightarrow y_{a}$ uniformly in $\mathbb{R} \times[0, T]$.

Proof. In view of (4.8) and Arzela's theorem there exist a subsequence $\left(h_{k}, \tau_{k}\right)_{k \in \mathbb{N}}$ with $\lim _{k \rightarrow \infty} h_{k}=0, \tau_{k} \leq \frac{h_{k}}{2 \beta}$ and a function $y \in C^{0}(\mathbb{R} \times[0, T])$, which is 2-periodic in space such that

$$
Y_{(k)}:=Y_{h_{k}, \tau_{k}} \rightarrow y \quad \text { uniformly in } \mathbb{R} \times[0, T] .
$$

We claim that $y$ is the viscosity solution of $(2.1)-(2.3)$, i.e., $y=y_{a}$. To see this, suppose that $\zeta \in C^{\infty}(\mathbb{R} \times(0, \infty))$ and that $y-\zeta$ has a local maximum at a point 
$\left(x_{0}, t_{0}\right) \in \mathbb{R} \times(0, T]$. Using standard arguments from the theory of viscosity solutions we may assume that this maximum is strict and global. In view of (4.9) there exists a sequence of gridpoints $\left(x_{j_{k}}, t_{n_{k}}\right) \in G_{h_{k}, \tau_{k}}$ such that $\lim _{k \rightarrow \infty}\left(x_{j_{k}}, t_{n_{k}}\right)=\left(x_{0}, t_{0}\right)$ and

$$
\xi_{k}:=Y_{(k), j_{k}}^{n_{k}}-W_{j_{k}}^{n_{k}}=\max _{G_{h_{k}, \tau_{k}}}\left(Y_{(k)}-W\right) .
$$

Here, $W_{j}^{n}=\zeta\left(x_{j}, t_{n}\right)$. In particular, $Y_{(k)}^{n_{k}-1} \leq W^{n_{k}-1}+\xi_{k}$ so that Lemma 4.1 implies

$$
\begin{aligned}
Y_{(k), j_{k}}^{n_{k}} & =\left(G\left(Y_{(k)}^{n_{k}-1}\right)\right)_{j_{k}} \leq\left(G\left(W^{n_{k}-1}\right)\right)_{j_{k}}+\xi_{k} \\
& =W_{j_{k}}^{n_{k}-1}+\tau_{k} a\left(x_{j_{k}}\right) \mathcal{F}\left(\delta_{h}^{+} W_{j_{k}}^{n_{k}-1}, \delta_{h}^{-} W_{j_{k}}^{n_{k}-1}\right),
\end{aligned}
$$

and hence, recalling the definitions of $W_{j}^{n}$ and $\xi_{k}$,

$$
\frac{\zeta\left(x_{j_{k}}, t_{n_{k}}\right)-\zeta\left(x_{j_{k}}, t_{n_{k}}-\tau_{k}\right)}{\tau_{k}} \leq a\left(x_{j_{k}}\right) \mathcal{F}\left(\delta_{h}^{+} W_{j_{k}}^{n_{k}-1}, \delta_{h}^{-} W_{j_{k}}^{n_{k}-1}\right) .
$$

Sending $k \rightarrow \infty$ we obtain $\zeta_{t}\left(x_{0}, t_{0}\right) \leq a^{*}\left(x_{0}\right) \sqrt{1+\zeta_{x}^{2}\left(x_{0}, t_{0}\right)}$, where we have used the smoothness of $\zeta$, the fact that $\lim _{k \rightarrow \infty}\left(x_{j_{k}}, t_{n_{k}}\right)=\left(x_{0}, t_{0}\right)$, and (4.7). Hence, $y$ is a viscosity subsolution of (2.1), and in a similar way one proves that $y$ is also a viscosity supersolution. Since the solution is unique, the whole sequence $\left(Y_{h, \tau}\right)$ converges to $y_{a}$. The relations (2.2) and (2.3) follow immediately from (4.9) and the definition of the scheme.

4.2. Discrete optimal control problem. In what follows we shall identify the function $a \in K$ with its values $a_{j}, j=1, \ldots, L$, and abbreviate $\underline{a}=\left(a_{1}, \ldots, a_{L}\right)^{t}$. We denote by $Y_{a}$ the solution of (4.2)-(4.4), where we always assume that $\tau \leq \frac{h}{2 \beta}$. In addition we suppose that the target $y_{T}$ satisfies $y_{T}(-1)=y_{T}(1)$ and denote by $Y^{T}$ the piecewise linear function on $I$ with values

$$
Y_{j}^{T}:=y_{T}\left(x_{j}\right), \quad j=0, \ldots, J .
$$

A discrete objective function is defined by

$$
\mathcal{J}_{h}(\underline{a}):=\frac{h}{2} \sum_{j=1}^{J}\left(Y_{a, j}^{N}-Y_{j}^{T}\right)^{2} .
$$

We consider the following discrete control problem:

$\left(\mathrm{P}_{h, \tau}\right) \quad$ minimize $\mathcal{J}_{h}(\underline{a})$, subject to $\underline{a} \in[\alpha, \beta]^{L}$.

Since the solution of the state equation depends continuously on $\underline{a}$ there exists a minimizer of the finite-dimensional optimization problem $\left(\mathrm{P}_{h, \tau}\right)$, which we denote by $\underline{a}_{h}^{*}$.

THEOREM 4.4. Suppose that $y_{T} \in H^{1}(I), y_{T}(-1)=y_{T}(1)$. There exists a sequence $h \rightarrow 0$ such that $\underline{a}_{h}^{*} \rightarrow \underline{a}^{*}$ and the associate function $a^{*} \in K$ is a minimum of $\mathcal{J}$.

Proof. Combining the bounds $\alpha \leq a_{h, j}^{*} \leq \beta$ with (4.8) we obtain a sequence $h \rightarrow 0$ and $\underline{a}^{*} \in[\alpha, \beta]^{L}, y \in C^{0}(\mathbb{R} \times[0, T])$, which is 2-periodic in space such that

$$
\underline{a}_{h}^{*} \rightarrow \underline{a}^{*}, \quad Y_{a_{h}^{*}} \rightarrow y \text { uniformly in } \mathbb{R} \times[0, T] .
$$

Copyright (C) by SIAM. Unauthorized reproduction of this article is prohibited. 
Similarly as in the proof of Theorem 4.3 it can be shown that $y=y_{a^{*}}$. Our aim is to prove that $\mathcal{J}\left(\underline{a}^{*}\right) \leq \mathcal{J}(\underline{a})$ for all $a \in K$. Since $\mathcal{J}_{h}\left(\underline{a}_{h}^{*}\right) \leq \mathcal{J}_{h}(\underline{a})$ for all $a \in K$ the claim follows, provided that we can show that

$$
\mathcal{J}_{h}(\underline{a}) \rightarrow \mathcal{J}(\underline{a}), \quad \mathcal{J}_{h}\left(\underline{a}_{h}^{*}\right) \rightarrow \mathcal{J}\left(\underline{a}^{*}\right)
$$

To begin, note that for a piecewise linear function $\eta: I \rightarrow \mathbb{R}$ with $\eta(-1)=\eta(1)$ we have

$$
\left|\int_{I} \eta^{2}-\frac{h}{2} \sum_{j=1}^{J}\left(\eta\left(x_{j-1}\right)^{2}+\eta\left(x_{j}\right)^{2}\right)\right| \leq C h^{2}\left\|\eta^{\prime}\right\|_{L^{2}(I)}^{2}
$$

Hence,

$$
\begin{aligned}
\left|\mathcal{J}(\underline{a})-\mathcal{J}_{h}(\underline{a})\right| & \leq C h^{2}\left\|Y_{a, x}^{N}-Y_{x}^{T}\right\|_{L^{2}(I)}^{2}+\frac{1}{2}\left|\left\|Y_{a}^{N}-Y^{T}\right\|_{L^{2}(I)}^{2}-\left\|y_{a}(\cdot, T)-y_{T}\right\|_{L^{2}(I)}^{2}\right| \\
& \leq C h^{2}\left(1+\left\|y_{T}\right\|_{H^{1}(I)}^{2}\right)+C\left(\left\|Y_{a}^{N}-y_{a}(\cdot, T)\right\|_{L^{2}(I)}+h\left\|y_{T}\right\|_{H^{1}(I)}\right) \\
& \rightarrow 0 \text { as } h \rightarrow 0,
\end{aligned}
$$

where we have used (4.8) and Theorem 4.3. Recalling (4.11), the second convergence in (4.12) can be shown in a similar way so that the result follows.

4.3. Adjoint equation. In order to compute the derivative of $\mathcal{J}_{h}$ with respect to $\underline{a}$ we formulate the following discrete adjoint equation. For fixed $\underline{a} \in K$ let $Y_{a}$ be as above. We then denote by $P: G_{h, \tau} \rightarrow \mathbb{R}$ the solution of the following backward problem:

$$
\begin{aligned}
P_{j}^{N}= & Y_{a, j}^{N}-Y_{j}^{T}, \quad j \in \mathbb{Z}, \\
-\delta_{t} P_{j}^{n+1}= & a\left(x_{j-1}\right) \frac{\partial \mathcal{F}}{\partial p}\left(\delta_{h}^{+} Y_{a, j-1}^{n}, \delta_{h}^{-} Y_{a, j-1}^{n}\right) P_{j-1}^{n+1}-a\left(x_{j}\right) \frac{\partial \mathcal{F}}{\partial p}\left(\delta_{h}^{+} Y_{a, j}^{n}, \delta_{h}^{-} Y_{a, j}^{n}\right) P_{j}^{n+1} \\
& +a\left(x_{j}\right) \frac{\partial \mathcal{F}}{\partial q}\left(\delta_{h}^{+} Y_{a, j}^{n}, \delta_{h}^{-} Y_{a, j}^{n}\right) P_{j}^{n+1}-a\left(x_{j+1}\right) \frac{\partial \mathcal{F}}{\partial q}\left(\delta_{h}^{+} Y_{a, j+1}^{n}, \delta_{h}^{-} Y_{a, j+1}^{n}\right) P_{j+1}^{n+1}, \\
(4.14) & j=1, \ldots, J, \quad n=N-1, \ldots, 0, \\
P_{j+J}^{n}= & P_{j}^{n}, \quad j \in \mathbb{Z} .
\end{aligned}
$$

Lemma 4.5. Let $a \in K$ and $P: G_{h, \tau} \rightarrow \mathbb{R}$ be the solution of (4.13), (4.14). Then

$$
\frac{\partial \mathcal{J}_{h}}{\partial a_{i}}(\underline{a})=h \tau \sum_{n=0}^{N-1} \sum_{j=1}^{J} \chi_{i j} \mathcal{F}\left(\delta_{h}^{+} Y_{a, j}^{n}, \delta_{h}^{-} Y_{a, j}^{n}\right) P_{j}^{n+1}, \quad i=1, \ldots, L
$$

where

$$
\chi_{i j}= \begin{cases}1 & \text { if } x_{j} \in\left(\hat{x}_{i-1}, \hat{x}_{i}\right) \\ \frac{1}{2} & \text { if } x_{j}=\hat{x}_{i-1} \text { or } x_{j}=\hat{x}_{i} \\ 0 & \text { otherwise }\end{cases}
$$

Copyright $@$ by SIAM. Unauthorized reproduction of this article is prohibited. 
Proof. Fix $i \in\{1, \ldots, L\}$. We define $Z: G_{h, \tau} \rightarrow \mathbb{R}$ by $Z_{j}^{n}=\frac{\partial Y_{a, j}^{n}}{\partial a_{i}}(\underline{a})$. In what follows we simply write $Y=Y_{a}$. Recalling (4.13) and observing that $Z_{j}^{0}=0$ we have

$$
\begin{aligned}
\frac{\partial \mathcal{J}_{h}}{\partial a_{i}}(\underline{a}) & =h \sum_{j=1}^{J}\left(Y_{j}^{N}-Y_{j}^{T}\right) Z_{j}^{N}=h \sum_{j=1}^{J} P_{j}^{N} Z_{j}^{N} \\
& =h \sum_{n=0}^{N-1} \sum_{j=1}^{J}\left(P_{j}^{n+1} Z_{j}^{n+1}-P_{j}^{n} Z_{j}^{n}\right) \\
& =h \sum_{n=0}^{N-1} \sum_{j=1}^{J}\left(P_{j}^{n+1}-P_{j}^{n}\right) Z_{j}^{n}+h \sum_{n=0}^{N-1} \sum_{j=1}^{J} P_{j}^{n+1}\left(Z_{j}^{n+1}-Z_{j}^{n}\right) .
\end{aligned}
$$

Differentiating (4.2) with respect to $a_{i}$ yields

$$
\begin{aligned}
\delta_{t} Z_{j}^{n}= & \chi_{i j} \mathcal{F}\left(\delta_{h}^{+} Y_{j}^{n}, \delta_{h}^{-} Y_{j}^{n}\right) \\
& +a\left(x_{j}\right) \frac{\partial \mathcal{F}}{\partial p}\left(\delta_{h}^{+} Y_{j}^{n}, \delta_{h}^{-} Y_{j}^{n}\right) \delta_{h}^{+} Z_{j}^{n}+a\left(x_{j}\right) \frac{\partial \mathcal{F}}{\partial q}\left(\delta_{h}^{+} Y_{j}^{n}, \delta_{h}^{-} Y_{j}^{n}\right) \delta_{h}^{-} Z_{j}^{n} .
\end{aligned}
$$

Hence from (4.16), (4.17), and (4.14) we obtain

$$
\begin{aligned}
\frac{\partial \mathcal{J}_{h}}{\partial a_{i}}(\underline{a})= & \tau \sum_{n=0}^{N-1} \sum_{j=1}^{J} Z_{j}^{n}\left\{-a\left(x_{j-1}\right) \frac{\partial \mathcal{F}}{\partial p}\left(\delta_{h}^{+} Y_{j-1}^{n}, \delta_{h}^{-} Y_{j-1}^{n}\right) P_{j-1}^{n+1}\right. \\
& +a\left(x_{j}\right) \frac{\partial \mathcal{F}}{\partial p}\left(\delta_{h}^{+} Y_{j}^{n}, \delta_{h}^{-} Y_{j}^{n}\right) P_{j}^{n+1} \\
& \left.-a\left(x_{j}\right) \frac{\partial \mathcal{F}}{\partial q}\left(\delta_{h}^{+} Y_{j}^{n}, \delta_{h}^{-} Y_{j}^{n}\right) P_{j}^{n+1}+a\left(x_{j+1}\right) \frac{\partial \mathcal{F}}{\partial q}\left(\delta_{h}^{+} Y_{j+1}^{n}, \delta_{h}^{-} Y_{j+1}^{n}\right) P_{j+1}^{n+1}\right\} \\
& +h \tau \sum_{n=0}^{N-1} \sum_{j=1}^{J} \chi_{i j} \mathcal{F}\left(\delta_{h}^{+} Y_{j}^{n}, \delta_{h}^{-} Y_{j}^{n}\right) P_{j}^{n+1} \\
& +\tau \sum_{n=0}^{N-1} \sum_{j=1}^{J} P_{j}^{n+1} a\left(x_{j}\right) \frac{\partial \mathcal{F}}{\partial p}\left(\delta_{h}^{+} Y_{j}^{n}, \delta_{h}^{-} Y_{j}^{n}\right)\left(Z_{j+1}^{n}-Z_{j}^{n}\right) \\
& +\tau \sum_{n=0}^{N-1} \sum_{j=1}^{J} P_{j}^{n+1} a\left(x_{j}\right) \frac{\partial \mathcal{F}}{\partial q}\left(\delta_{h}^{+} Y_{j}^{n}, \delta_{h}^{-} Y_{j}^{n}\right)\left(Z_{j}^{n}-Z_{j-1}^{n}\right) \\
= & h \tau \sum_{n=0}^{N-1} \sum_{j=1}^{J} \chi_{i j} \mathcal{F}\left(\delta_{h}^{+} Y_{j}^{n}, \delta_{h}^{-} Y_{j}^{n}\right) P_{j}^{n+1}
\end{aligned}
$$

by shifting indices and using the periodicity of $Z$ and $P$.

4.4. Optimization method. We set $P_{S}(\underline{a})_{i}=\max \left(\alpha, \min \left(a_{i}, \beta\right)\right)$ for $i=1,2$, $\ldots, L$ and now define a projected gradient algorithm to solve the problem $\left(\mathrm{P}_{h, \tau}\right)$.

Step 1 Choose $\underline{a}^{0} \in[\alpha, \beta]^{L}, \gamma \in(0,1)$ and tol.

Step 2 For $k=0,1,2, \ldots$, do Steps $3-6$.

Step 3 Set $s^{k}=-\nabla \mathcal{J}_{h}\left(\underline{a}^{k}\right)=-\left(\frac{\partial \mathcal{J}_{h}}{\partial a_{1}}\left(\underline{a}^{k}\right), \ldots, \frac{\partial \mathcal{J}_{h}}{\partial a_{L}}\left(\underline{a}^{k}\right)\right)$.

Step 4 Choose the minimum $\sigma_{k} \in\left\{1, \frac{1}{2}, \frac{1}{4}, \ldots\right\}$ for which

$$
\mathcal{J}_{h}\left(P_{S}\left(\underline{a}^{k}+\sigma^{k} s^{k}\right)\right)-\mathcal{J}_{h}\left(\underline{a}^{k}\right) \leq-\frac{\gamma}{\sigma_{k}}\left\|P_{S}\left(\underline{a}^{k}+\sigma^{k} s^{k}\right)-a^{k}\right\|_{2}^{2} .
$$

Copyright (c) by SIAM. Unauthorized reproduction of this article is prohibited. 

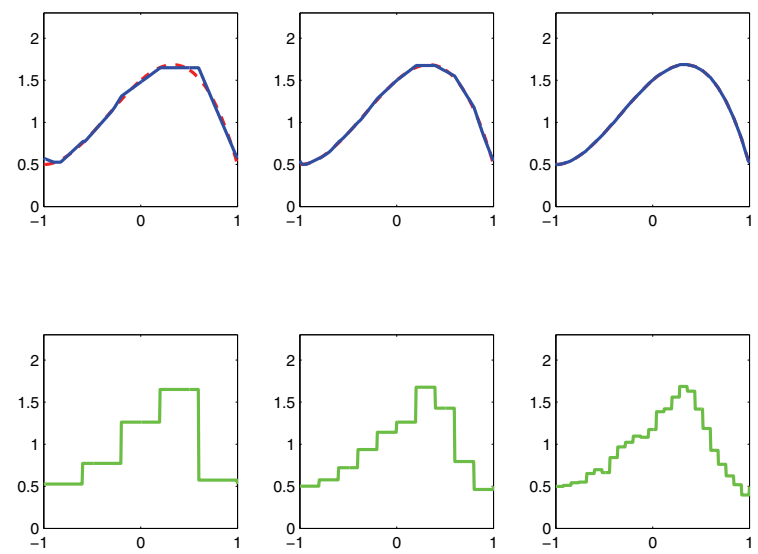

FIG. 4.1. Results for (4.18); upper plots show $Y^{T}$ (dashed line) and $Y_{a}^{N}$ (solid line), and lower plots show $\underline{a}$.

Step 5 Set $\underline{a}^{k+1}=P_{S}\left(\underline{a}^{k}+\sigma_{k} s^{k}\right)$.

Step 6 If $\left\|\underline{a}^{k+1}-\underline{a}^{k}\right\|_{2}<$ tol, then STOP.

Here, $\|\cdot\|_{2}$ denotes the Euclidean norm in $\mathbb{R}^{L}$.

4.5. Numerical experiments. We consider four numerical experiments, using the following values of $y_{T}(x)$ :

$$
\begin{aligned}
& y_{T}(x)=0.5+\left(1-x^{2}\right)(1+x), \\
& y_{T}(x)=\left\{\begin{array}{cc}
2.0, & -1 \leq x \leq-0.5, \\
2.0-3\left|x+\frac{1}{2}\right|, & -0.5 \leq x \leq 0, \\
2.0-3\left|x-\frac{1}{2}\right|, & 0 \leq x \leq 0.5 \\
2.0, & 0.5 \leq x \leq 1
\end{array}\right. \\
& y_{T}(x)=\left\{\begin{array}{cc}
1+\sqrt[3]{-x}, & -1 \leq x \leq 0 \\
1+\sqrt{x}, & 0<x \leq 1
\end{array}\right. \\
& y_{T}(x)=\left\{\begin{array}{cc}
0.5, & -1 \leq x<-0.25 \\
2.0, & -0.25 \leq x \leq 0.25, \\
0.5, & 0.25<x \leq 1
\end{array}\right.
\end{aligned}
$$

In all our computations we take $T=1, \alpha=0.1, \beta=4, h=0.005, \tau=\frac{h}{2 \beta}$, tol $=10^{-9}$, and $\gamma=0.1$. Then for each of the four experiments we take $L=5,10$, and 25 .

The computational results for $y_{T}$ given by (4.18) are displayed in Figure 4.1. We see six subplots: The upper three plots show $Y^{T}$ (dashed line) and $Y_{a}^{N}$ (solid line), while the lower three plots show $a$. In the left-hand plots we took $L=5$, in the center plots we took $L=10$, and in the right-hand plots we took $L=25$. Figure 4.2 takes the same form as Figure 4.1 except that (4.19) was used in place of (4.18).

In both these examples the target $y_{T}$ is Lipschitz and so in principle is attainable. On the other hand the target in each of the next two examples is not Lipschitz.

Copyright (c) by SIAM. Unauthorized reproduction of this article is prohibited. 

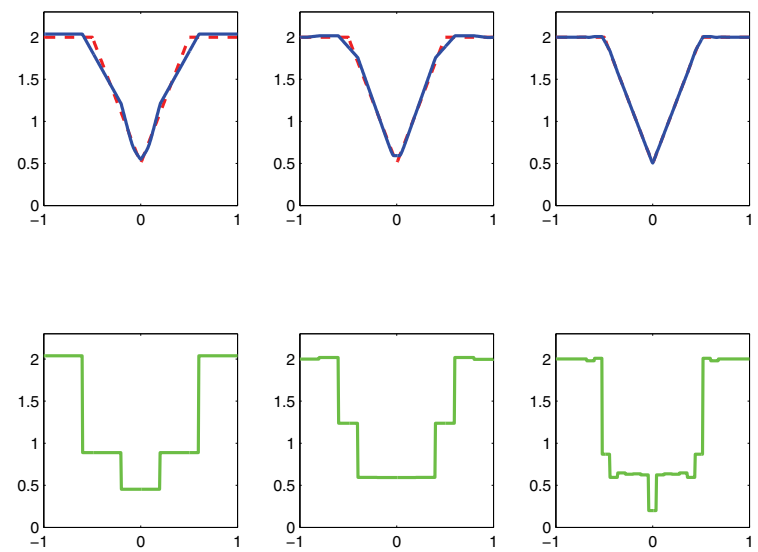

FIG. 4.2. Results for (4.19); upper plots show $Y^{T}$ (dashed line) and $Y_{a}^{N}$ (solid line), and lower plots show $\underline{a}$.
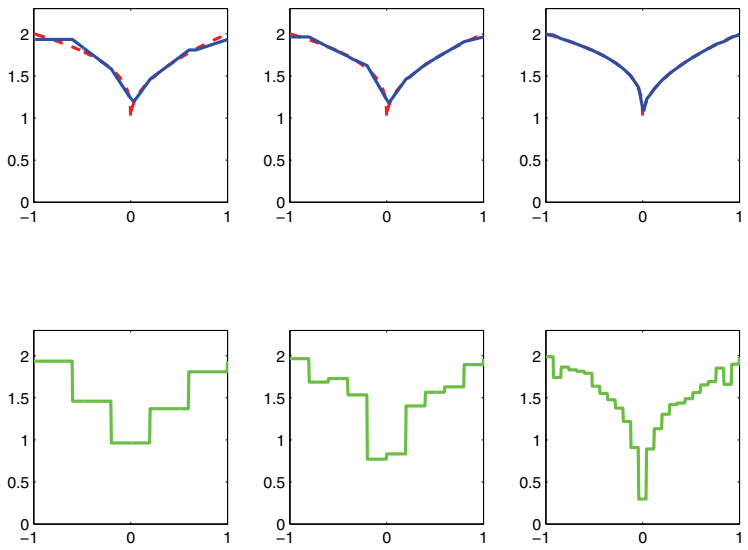

FIG. 4.3. Results for (4.20); upper plots show $Y^{T}$ (dashed line) and $Y_{a}^{N}$ (solid line), and lower plots show $\underline{a}$.

Figures 4.3 and 4.4 take the same form as Figure 4.1 except that (4.20) and (4.21) were used in place of (4.18). In Table 4.1 we show the value of the discrete objective functional $\mathcal{J}_{h}(\underline{a}):=\frac{h}{2} \sum_{j=1}^{J}\left(Y_{a, j}^{N}-Y_{j}^{T}\right)^{2}$ for the computations described above. Note that the target in each of the first three examples satisfies the conditions in Theorem 3.2 , whereas the final example does not. This is reflected in the values of the objective functional.

We now turn to the performance of the iterative scheme. For the results in Figures 4.1 and 4.2 we took $a_{i}^{0}=\alpha=0.1, i=1,2, \ldots, L$; however, we also ran both computations with different initial data for $\underline{a}$, in particular $a_{i}^{0}=\beta=4$, and $a_{i}^{0}=0.5(\alpha+\beta)=2.05, i=1,2, \ldots, L$. In both examples the iteration count did not vary significantly so that in the absence of other information we simply set $a_{i}^{0}=$ $\alpha=0.1, i=1,2, \ldots, L$, in the remaining computations. In Table 4.2 we show the CPU time (on an Intel Core2 Extreme Processor with $3.0 \mathrm{GHz}$ clock speed and $6 \mathrm{MB}$ RAM).

We conclude with Figures 4.5 and 4.6 in which we look at the convergence of 

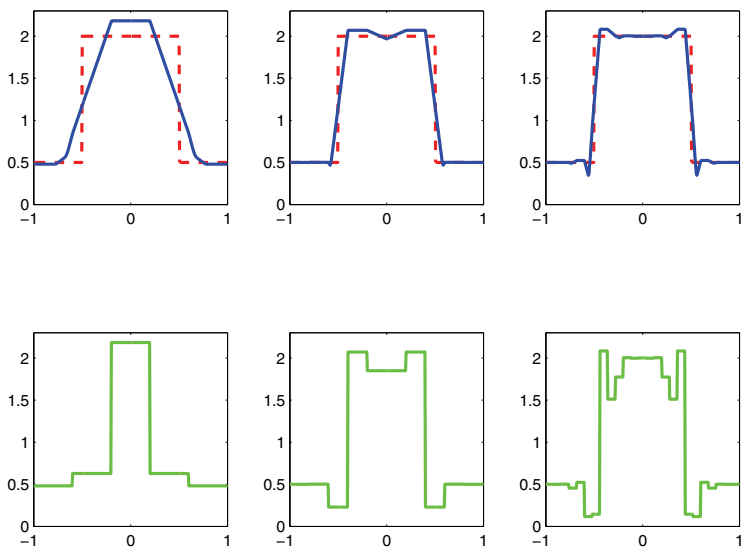

FIG. 4.4. Results for (4.21); upper plots show $Y^{T}$ (dashed line) and $Y_{a}^{N}$ (solid line), and lower plots show $\underline{a}$.

TABLE 4.1 $\mathcal{J}_{h}(\underline{a})$.

\begin{tabular}{|c||c|c|c|}
\hline & $L=5$ & $L=10$ & $L=25$ \\
\hline \hline$(4.18)$ & $1.036 e^{-2}$ & $1.015 e^{-4}$ & $5.976 e^{-6}$ \\
\hline$(4.19)$ & $4.071 e^{-2}$ & $1.134 e^{-2}$ & $4.282 e^{-5}$ \\
\hline$(4.20)$ & $1.582^{-2}$ & $4.923 e^{-4}$ & $6.702 e^{-6}$ \\
\hline$(4.21)$ & $9.382 e^{-2}$ & $3.449 e^{-2}$ & $2.097 e^{-2}$ \\
\hline
\end{tabular}

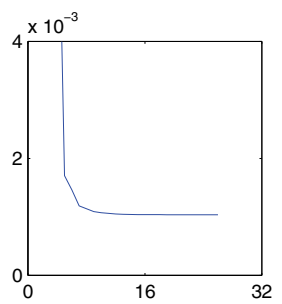

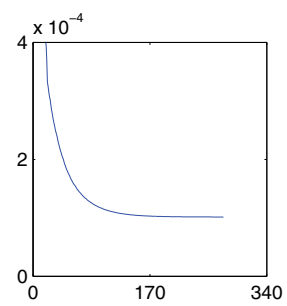

TABLE 4.2

CPU time.

\begin{tabular}{|c||c|c|c|}
\hline & $L=5$ & $L=10$ & $L=25$ \\
\hline \hline$(4.18)$ & 14.96 & 178.19 & 780.19 \\
\hline$(4.19)$ & 22.89 & 241.41 & 727.70 \\
\hline$(4.20)$ & 66.04 & 405.12 & 1021.77 \\
\hline$(4.21)$ & 12.76 & 580.98 & 1274.79 \\
\hline
\end{tabular}

FIG. 4.5. Plots of $\mathcal{J}_{h}\left(\underline{a}^{k}\right)$ for $L=5$ (left), $L=10$ (center), and $L=25$ (right) with $y_{T}$ given by (4.18).
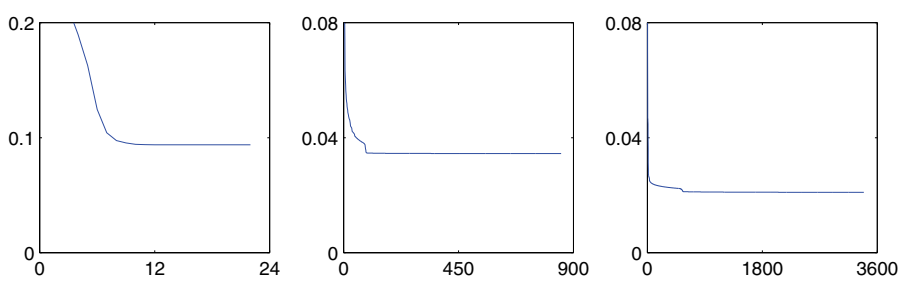

FIG. 4.6. Plots of $\mathcal{J}_{h}\left(\underline{a}^{k}\right)$ for $L=5$ (left), $L=10$ (center), and $L=25$ (right) with $y_{T}$ given by $(4.21)$.

$\mathcal{J}_{h}\left(\underline{a}^{k}\right)$ as $k$ increases; in Figure 4.5 we took $y_{T}$ given by (4.18) and compared the value of $\mathcal{J}_{h}\left(\underline{a}^{k}\right)$, with $k$ large, for three values of $L ; L=5,10$, and 25. Each plot shows $\mathcal{J}_{h}\left(\underline{a}^{k}\right)$ plotted against the iteration number $k$; the left-hand plot displays results for

Copyright (c) by SIAM. Unauthorized reproduction of this article is prohibited. 
$L=5$, the center plot for $L=10$, and the right-hand plot for $L=25$. Figure 4.6 takes the same form as Figure 4.5 except that (4.21) was used in place of (4.18). From Figures 4.5 and 4.6 we see that for each value of $L, \mathcal{J}_{h}\left(\underline{a}^{k}\right)$ converges, say to $\mathcal{J}_{h}\left(\underline{a}^{*}\right)$, and that as $L$ increases $\mathcal{J}_{h}\left(\underline{a}^{*}\right)$ decreases.

5. Conclusion. We have formulated an optimal control problem in which the final shape of a one-dimensional graph evolving with a prescribed inhomogeneous speed is controlled by varying the speed function. The problem is one of controlling a first order Hamilton-Jacobi equation. The mathematical formulation is one of a quadratic objective functional with a state equation which is posed in the sense of viscosity solutions. Existence is shown for several variants for constraints on the speed function. An approximate controllability result in the $\left(H^{1}\right)^{\prime}$-norm is proved with respect to the number of intervals defining a piecewise constant speed function. A discrete version of the control problem was formulated and shown to be convergent as the mesh size goes to zero. Our numerical method was based on a scheme for the state equation which was differentiable with respect to the discrete state. This allowed the derivation of an adjoint equation. Finally we displayed some numerical results.

This is an example of an optimal control problem in which the nonlinear state equation is not formulated in a classical manner but in the sense of viscosity solutions and in which the solution of the state equation is not differentiable with respect to the control variable. The approach of this article could be extended to consideration of higher space dimensional problems.

\section{REFERENCES}

[1] G. Barles And P. E. Souganidis, Convergence of approximation schemes for fully nonlinear second order equations, Asymptotic Anal., 4 (1991), pp. 271-283.

[2] J. M. Berg, A. Yezzi, And A. R. Tannenbaum, Phase transitions, curve evolution and the control of semiconductor manufacturing processes, in Proceedings of the IEEE Conference on Decision and Control, Kobe, Japan, 1996, pp. 3376-3381.

[3] J. M. Berg AND N. Zhou, Shape-based optimal estimation and design of curve evolution processes with application to plasma etching, IEEE Trans. Automat. Control, 46 (2001), pp. $1862-1873$.

[4] C. Castro, F. Palacios, and E. Zuazua, An alternating descent method for the optimal control of the inviscid Burgers equation in the presence of shocks, Math. Models Methods Appl. Sci., 18 (2008), pp. 369-416.

[5] M. G. Crandall and P. L. Lions, Viscosity solutions of Hamilton-Jacobi equations, Trans. Amer. Math. Soc., 277 (1983), pp. 1-42.

[6] M. G. Crandall, L. C. Evans, and P. L. Lions, Some properties of viscosity solutions of Hamilton-Jacobi equations, Trans. Amer. Math. Soc., 282 (1984), pp. 487-502.

[7] K. Deckelnick and C. M. Elliott, Uniqueness and error analysis for Hamilton-Jacobi equations with discontinuities, Interfaces Free Bound., 6 (2004), pp. 329-349.

[8] K. Deckelnick And C. M. Elliott, Propagation of graphs in two-dimensional inhomogeneous media, Appl. Numer. Math., 56 (2006), pp. 1163-1178.

[9] H. IsHII, Hamilton-Jacobi equations with discontinuous Hamiltonians on arbitrary open sets, Bull. Fac. Sci. Engrg. Chuo. Univ., 28 (1985), pp. 33-77.

[10] O. A. Ladyzhenskaya, V. A. Solonnikov, and N. N. Uralceva, Linear and Quasilinear Equations of Parabolic Type, Transl. Math. Monogr. 24, AMS, Providence, RI, 1968.

[11] S. LEUng AND J. QIAN, An adjoint state method for three-dimensional transmission traveltime tomography using first-arrivals, Commun. Math. Sci., 4 (2006), pp. 249-266.

[12] J. A. Sethian, Level Set Methods, Cambridge University Press, Cambridge, UK, 1996.

[13] S. UlbRich, A sensitivity and adjoint calculus for discontinuous solutions of hyperbolic conservation laws with source terms, SIAM J. Control Optim., 41 (2002), pp. 740-797. 